\title{
9 Die Pendelbewegung des Übergangs: Die Architektur des Scheins
}

Gerade Hegels Überlegungen zum „Schein“ sind für unsere Fragestellung bereits in jeder Hinsicht ,wesentlich“ und mehr als eine bloße Brücke zur ,eigentlichen` Entwicklung der Reflexionsformen des Wesens. Bezeichnenderweise ist zudem das gesamte „Erste Kapitel“ des „Ersten Abschnitts“ der Wesenslogik, um das es im Folgenden vor allem gehen wird, mit dem Begriff des „Scheins“ bezeichnet und so eine Teilüberschrift („B. Der Schein“) zugleich zur Hauptüberschrift des ganzen Kapitels gemacht. Bereits damit ist angedeutet, dass die Bedeutsamkeit des Scheins weit darüber hinausgeht, bloß gewissermaßen das letzte Aufglimmen der Seinslogik vor ihrem Verlöschen im Bereich des Wesens zu sein. Hegels Überlegungen zum Schein sind aber nicht nur strukturell, sondern auch intrinsisch schwierig: weil sie zwar an die wichtigsten klassischen Interpretamente der (neu) platonischen und späterhin vor allem ästhetischen Theoriegeschichte des Scheins anschließen - in der Dopplung von Schein als Trug/Täuschung und zugleich Erscheinung, Auftauchen bzw. Offenbaren des Wahren ${ }^{65}$-, zugleich aber ein deutliches Komplexerwerden der darin ausgedrückten Gedanken darstellen, das nicht nur mit der Eingliederung dieser Ideen in die argumentative Architektur der Logik zusammenhängt. Im Begriffszusammenhang des Scheins denkt Hegel nämlich darüber nach, wie sich in der Logik des Wesens architektonische Bestimmungen der Sphäre des Seins auch dann erhalten, wenn sie für sich genommen nicht mehr haltbar sind: wie also die logische Bilanz des Übergangs von der Seins- zur Wesenslogik aussieht und welche dabei festgestellten Bestände trotz ihrer Überwindung zugleich unveräußerlich in einem starken Sinne bleiben. Dieser starke Sinn des Überdauerns ist dabei nicht dasselbe wie das Moment des conservare im Modus der „Aufhebung“66: Es geht also in ihm nicht bloß darum

65 Vgl. Iber: Metaphysik absoluter Relationalität, S. 68.

66 „Unter aufheben verstehen wir einmal soviel als hinwegräumen, negieren, und sagen demgemäß z.B., ein Gesetz eine Einrichtung usw. seien aufgehoben. Weiter heißt dann aber auch aufheben soviel als aufbewahren, und wir sprechen in diesem Sinn davon, daß etwas wohl aufgehoben sei. Dieser sprachgebräuchliche Doppelsinn, wonach dasselbe Wort eine negative und eine positive Bedeutung hat, darf nicht als zufällig angesehen noch etwa gar der Sprache zum Vorwurf gemacht werden, als zu Verwirrung Veranlassung gebend, sondern es ist darin der über das bloß verständige Entweder-Oder hinausschreitende spekulative Geist unserer Sprache zu erkennen.“ (TWA 8, S. 204 f., § 96, [Zusatz]) Sowie: „A u f he ben und das Aufg e hob ene (das Ideelle) ist einer der wichtigsten Begriffe der Philosophie, eine Grundbestimmung, die schlechthin allenthalben wiederkehrt, deren Sinn bestimmt aufzufassen und besonders vom Nichts zu unterscheiden ist. - Was sich aufhebt, wird dadurch nicht zu Nichts. Nichts ist das 
festzustellen, wie das auf bestimmte Weise negierte Sein in der Transformation von äußeren Elementen eines Gegensatzes zu inneren Momenten einer Begriffseinheit im Wesen frühere Inhalte und Funktionsgehalte auch bewahrt. Gerade am Schein wird nämlich deutlich, wie Hegel die Notwendigkeit einer zumindest temporären Perennienz eines eigentlich bereits Aufgehobenen als Nicht-Aufgehobenes denkt - und aus welchen Gründen dies für bestimmte Argumentationszusammenhänge wichtig sein könnte. Am Element des „Scheins“ wird so deutlich, dass Hegels Entfaltung der je konkreten Bewegungsbedingungen des Begriffs in der Logik komplexer, vielgestaltiger und für bestimmte Problemlösungen individueller ist, als es die Explikation des allgemeinen Schemas dialektischer Aufhebung und bestimmter Negation (GW 20, S. 118-120, §§ 79-83) nahelegt: selbst dann, wenn man dies jeweils noch nach bestimmten Arten, d.h. besonderen Funktionsgehalten von Dialektik (Übergangsdialektik, Reflexionsdialektik, Entwicklungsdialektik), bezogen auf die Teile der Logik, ausdifferenziert. ${ }^{67}$

Dabei zeichnet Hegel diesen Anfang des Prozesses des Übergangs zwischen Sein und Wesen als eine Art Pendelbewegung zwischen beiden aus, welche die grundsätzlich monodirektionale Transformationsrichtung vom Sein zum Wesen im Strukturgesetz der aufhebenden Entwicklung (das Sein hebt sich im Wesen auf) als zugleich bidirektionales Widerspiel - und damit selbst als genuine Reflexionsbewegung des Hin und Her - vollzieht. Im Fortgang vom Sein zum Wesen stellen sich nämlich zu Anfang rhythmisch ${ }^{68}$ Seinsbestände rückfallartig wieder her und verweisen zugleich in der Wiederherstellung auf ihre erfolgte und noch zu erfolgende Aufhebung im Wesen. Dabei folgt diese Bewegung dem Sich-Ereignen eines (wenn man so will) doppelten, zueinander inversen Erinnerungsgeschehens, das wiederum mit bestimmten logischen, d.h. subjektlosen Mechanismen von Verdrängung und Wiederkehr zu arbeiten scheint: Die aufhebende Reformulierung der Bestimmungen des Seins nach der Grammatik des Wesens bringt das

\footnotetext{
Unmittelbare; ein Aufgehobenes dagegen ist ein Vermitteltes, es ist das Nichtseyende, aber als Resultat, das von einem Seyn ausgegangen ist; es hat daher die Bestimmtheit, aus der es herkommt, noch an sich. Aufheben hat in der Sprache den gedoppelten Sinn, daß es soviel als aufbewahren, er h a l t en bedeutet und zugleich soviel als aufhören lassen, ein Ende machen." (GW 21, S. 94, Anm.)

67 Schäfer: Die Dialektik und ihre besonderen Formen, S. 295-329. Vgl. TWA 8, S. 308, § 161 [Zusatz].

68 Wie wichtig für Hegel, auch im Sinne philosophischen Zusammenhangs, der „Rhythmus“ ist, zeigt sich bereits in der „Vorrede“ zur Phänomenologie des Geistes: „In dieser Natur dessen, was ist, in seinem Seyn sein Begriff zu seyn, ist es, daß überhaupt die lo g i s c he N o th w en dig ke it besteht; sie allein ist das vernünftige und der Rhythmus des organischen Ganzen.“ (GW 9, S. 40) Es sei sodann auch die „Natur der wissenschaftlichen Methode“, welche „selbst ihren Rhythmus zu bestimmen“ hat (GW 9, 41).
} 
„zeitlos vergangene Seyn“ (GW 11, S. 241) und seine möglicherweise erhaltenswerten Dimensionen in das logische Gedächtnis des Fortgangs und führt zum Sein zurück; zugleich aber fordert im Rückgang auf die Bedingungen des Seins die bereits erfolgte Transfiguration des Wesens wiederum erinnernd ihre unrevidierbare Stellung und damit die nicht mehr rücknehmbaren Fortschritte der logischen Bewegung ein. ${ }^{69}$ Argumentationslogisch ist es eben dieses pendelartige Widerspiel, das zusätzlich zu allen Einzelargumenten die Integration des Seins in das Wesen, d. h. die neue Ebene der Unmittelbarkeit des Wesens, nicht nur erklärt, sondern sozusagen performativ inszeniert, indem in der dichten Bewegung des Vor und Zurück die Grenze zwischen Sein und Wesen fällt, quasi überspielt wird. Die Inszenierung dieser Pendelbewegung vollzieht sich dabei an Überlegungen, welche zu Beginn der Wesenslogik (Teil A und B) zunächst einmal ausnahmslos das Verhältnis der ganzen Sphäre des Seins zur ganzen Sphäre des Wesens betreffen, also das jeweils Allgemeine von Sein und Wesen zueinander in Beziehung setzen und die Stadien ihres Übergangs reflektieren. Die Ebene einer solchen Allgemeinheit und damit das Nachdenken über das Verhältnis der logischen Grammatiken von Sein und Wesen überhaupt - d.h. die Reflexion der wesentlichen, resultativen wie tragenden allgemeinen Bestimmungen der metaphysischen Syntax von Sein und Wesen - ermöglicht es Hegel dabei, im makrostrukturellen Blick auf den Raum der logischen Großsphären den Verlauf der je einzelnen Reformulierungen der seinslogischen Bestimmungen anzuhalten, um stattdessen kategoriale Formationen zweiter Ordnung scharf zu stellen. Eben die aber werden im Folgenden die Möglichkeit bereitstellen, jenseits bloß einzelner Kategorien der Logik die minimale Realitätskategorie als dazu hyperformative Struktur zu reflektieren. Damit wird aus der kantkritischen Einsicht, dass sich die fundamentale Kategorie des Realen nicht auf eine isolierte Position auf der Kategorientafel beschränken lässt, sondern einer solchen Tafel vielmehr noch zuvor liegt bzw. quer über diese verläuft, die notwendige Konsequenz gezogen.

69 Es scheint mir eine bisher nicht hinreichend untersuchte Dimension sowohl des Gangs der Phänomenologie des Geistes als auch der Wissenschaft der Logik zu sein, die mit ihrem Entwicklungskonzept des Gegenstandes (Geist - absolute Idee) und den dichten zyklischen Vor- und Rückverweisen ihres Vorgehens verbundende Idee einer logischen Erinnerung und logischen Erwartung in ihrer Struktur und Funktion für das jeweilige Werk bzw. für das System überhaupt in den Blick zu nehmen. Die Frage, ob Hegels System und Systemteile logische Arten der Protention und Retention kennen, wie diese funktionieren, welchen Stellenwert sie für den jeweiligen Fortgang spielen und inwiefern sie vielleicht noch unentdeckte Problembeschreibungs- und Problemlösungspotenziale enthalten, scheint mir in jedem Fall eine lohnende zu sein, der hier aber nicht weiter nachgegangen werden kann. 